\title{
Racl overexpression is correlated with epithelial mesenchymal transition and predicts poor prognosis in non-small cell lung cancer
}

\author{
Yujuan Zhou*, Qianjin Liao*, Yaqian Han, Jie Chen, Zhigang Liu, Hang Ling, Jing Zhang, Wenjuan Yang, \\ Linda Oyang, Longzheng Xia, Li Wang, Heran Wang, Lei Xue, Hui Wang ${ }^{\bowtie}$, Bingqiang $\mathrm{Hu}^{凶}$
}

Key Laboratory of Translational Radiation Oncology, Hunan Province, Hunan Cancer Hospital and The Affiliated Cancer Hospital of Xiangya School of Medicine, Central South University, 283 Tongzipo Road, Changsha 410013, Hunan, China.

*These authors contributed equally to this work.

$\triangle$ Corresponding authors: Hui Wang and Bingqiang Hu, Key Laboratory of Translational Radiation Oncology, Hunan Province, Hunan Cancer Hospital and The Affiliated Cancer Hospital of Xiangya School of Medicine, Central South University, 283 Tongzipo Road, Changsha 410013, Hunan, China. Tel: 86-731-88651680; Fax: 86-731-88651999; Email: wanghui710327@163.com, hubingqiang@ hnszlyy.com.

๑ Ivyspring International Publisher. Reproduction is permitted for personal, noncommercial use, provided that the article is in whole, unmodified, and properly cited. See http://ivyspring.com/terms for terms and conditions.

Received: 2016.05.17; Accepted: 2016.08.14; Published: 2016.10.23

\begin{abstract}
Objective: Ras-related C3 botulinum toxin substratel (Racl) and epithelial mesenchymal transition (EMT) are key therapeutic targets in cancer. We investigated the clinical significance of Racl and markers of EMT expression in non-small cell lung cancer (NSCLC), and their possible correlation with EMT phenotype.

Methods: Immunohistochemistry was used to assess the expression of Racl, Snaill, Twistl, $\mathrm{N}$-cadherin (N-cad), Vimentin (Vim), and E-cadherin (E-cad) in 153 NSCLC paraffin-embedded specimens and 45 normal specimens adjacent to tumors. The correlation of Racl and EMT markers with clinicopathological characteristics and the relationship between the protein levels and progression-free survival (PFS) and overall survival (OS) were analyzed.

Results: Compared with non-tumor tissues, the NSCLC tissues showed marked elevation in the levels of Racl, Snaill, Twist I, N-cad, and Vim levels, whereas the E-cad levels were significantly decreased (P $<0.05)$. The aberrant expression of Racl and EMT markers was significantly associated with TNM stage and metastasis $(P<0.05)$. Increased expression of Racl may be associated with poor OS and PFS compared with low expression $(P<0.001$ and $P=0.004)$. Significant correlations were observed between the EMT markers expressed and OS or PFS $(P<0.01)$. In addition, multivariate analysis indicated that the expression of Racl, Snaill, Twistl, N-cad, Vim, and E-cad was an independent prognostic factor in NSCLC. Interestingly, Racl expression was positively correlated with Snaill, Twistl, N-cad, and Vim levels ( $r=0.563, r=0.440, r=0.247 r=0.536, P<0.01$, respectively) and negatively correlated with $E$-cad levels $(r=-0.464, P<0.001)$ in NSCLC tissues. Racl, Twist, Snaill, Vim and N-cad were highly expressed in lung cancer patients resistant to radiotherapy, while E-cad was poorly expressed.

Conclusion: Racl may promote NSCLC progression and metastasis via EMT, which may be considered as a potential therapeutic target.
\end{abstract}

Key words: non-small cell lung cancer; Rac1; epithelial-mesenchymal transition; prognosis; markers; radiotherapy.

\section{Introduction}

Lung cancer is the most prevalent human malignancy with the highest incidence and mortality rates [1]. Non-small-cell lung cancer (NSCLC) is the predominant form of lung cancer, which includes adenocarcinoma and squamous cell carcinoma [2]. Despite recent advances in clinical and experimental oncology, the prognosis of patients with NSCLC is still poor with a 5-year survival rate of about $15 \%$ [3], 
mainly due to invasion and metastasis. Therefore, elucidation of the molecular mechanisms underlying metastasis and tumor progression is essential for the development of treatment strategies. Patients may benefit from molecular therapy, which specifically targets biological markers of metastases and tumor progression.

Epithelial-mesenchymal transition (EMT) is a reversible embryonic process and is abnormally activated in tumor progression, metastasis and resistance to chemoradiotherapy [4]. Activation of EMT is associated with altered expression of many genes, including down-regulation of epithelial markers such as E-cadherin (E-cad) and zonula occluden-1 (ZO-1); up-regulation of mesenchymal marker genes, including $\mathrm{N}$-cadherin $(\mathrm{N}$-cad) and Vimentin (Vim), and transcription factors or activation of Snail and Twist [5]. Many studies have shown that EMT is a critical process in tumor invasion and metastatsis in NSCLC [6-8]. Studies found that ionizing radiation (IR) increases resistance to radioactivity by inducing EMT changes in tumor cells, and promotes tumor relapse and metastasis $[4,9,10]$. However, the underlying mechanism still needs to be elucidated.

TGF- $\beta$ /Smad, Ras/MAPK, Notch and Wnt $/ \beta$-catenin signal transduction pathways regulate the EMT [11]. Recent studies involving Rac GTP enzyme (Rho protein family member) regulation of EMT in tumor cells attracted much concern [12-14]. Ras-related C3 botulinum toxin substrate1(Rac1) plays a key role in regulating the recombination of cytoskeletal proteins as well as malignant transformation, adhesion, migration, invasion, proliferation, differentiation and apoptosis of tumor cells [15]. Similar to most of the GTP enzymes, the biological function of Rac1 depends on activation and inactivation. After Rac1 activation, the signal is transmitted to down-stream p21-activating kinase (Pak 1) [15] that regulates the balance between phosphorylation and de-phosphorylation of actin depolymerizing factor (ADF/Cofilin) and cytoskeletal reorganization, which further promotes EMT [16]. Rac1 is over-expressed in many tumors, and is closely associated with tumor invasion [17]. The Rac1 expression level is positively correlated with tumor progression, metastases and poor prognosis of specific carcinomas [18]. Inhibition of Rac1 expression or disruption of its function may significantly inhibit cancer cell proliferation and metastasis [17, 19]. Studies show that Rho GDI2 up-regulated the expression of epithelial marker E-cad by inhibiting Rac1expression, down-regulated the expression of Slug, Snail and a-SMA, and further inhibited EMT changes in lung cancer cells [20]. However, the expression and correlation of Rac1 and EMT molecular markers in lung cancer specimens as well as resistance to radiation requires further investigation. In our study, we found that the expression of Rac1 and EMT was abnormal. The expression of Rac1 and EMT markers showed significant correlation with TNM stage, metastasis, prognosis, and radiotherapy resistance. Rac1 and EMT markers are potential molecular markers for prognosis and efficacy monitoring in lung cancer.

\section{Materials and methods}

\section{Tumor samples}

Lung cancer samples (153 cases) and normal tissues adjacent to the cancer site (45 cases) were excised from patients admitted to the Hunan Cancer Hospital/The Affiliated Cancer Hospital of Xiangya School of Medicine, Central South University (China) during 2002 to 2004. A follow-up survival analysis was conducted after four to 95 months, over an average follow-up period of 57 months. The clinicopathological characteristics are listed in Table 1. All the patients were selected and treated with radiotherapy with a total cumulative dose ranging from 40-66 Gy, with 2 Gy per fraction administered over 5 days per week after surgery. None of the patients received any neoadjuvant therapy. They were all diagnosed pathologically. Patients' name, sex, age, pathological diagnosis, tumor, nodal status, metastasis and TNM classification were recorded.

As previously described [21], the main criterion of grouping was overall survival, calculated from the date of radiotherapy to the time of death (from any cause). The secondary criterion was local or distant recurrence rate. Local recurrence was defined as cancer growth in the same lung or in the mediastinum. A new peripheral contralateral lesion was classified as distant recurrence. According to these criteria, the patients were divided into two groups: radiotherapy sensitive $(54,35.3 \%)$ and resistant $(99,64.7 \%)$.

\section{Immunohistochemistry (IHC)}

IHC studies were performed using the standard streptavidin/peroxidase staining method, as described previously [22]. The following antibodies were used: anti-Rac1(1:1000), anti-Snail1(1:200), anti-N-cad (1:500), anti-Twist1(1:1000) (Abcam, Cambridge, UK), anti-E-cad, and anti-Vim (Maxin, Fuzhou, China). All known positive sections served as positive controls. Negative mouse serum and PBS were used instead of the first antibody as a negative control and blank control, respectively. 
Table 1. Relationship between Racl expression and clinical pathology.

\begin{tabular}{|c|c|c|c|c|}
\hline \multirow[t]{2}{*}{ Variable } & \multirow[t]{2}{*}{$\mathrm{N}$} & \multicolumn{3}{|c|}{ Rac1 } \\
\hline & & High & Low & $P$ Value \\
\hline \multicolumn{5}{|l|}{ Age (years) } \\
\hline$\leq 60$ & 57 & 39 & 18 & \\
\hline$>60$ & 96 & 72 & 24 & 0.378 \\
\hline \multicolumn{5}{|l|}{ Gender } \\
\hline Male & 141 & 103 & 38 & 0.634 \\
\hline female & 12 & 8 & 4 & \\
\hline \multicolumn{5}{|l|}{ Smoking } \\
\hline Yes & 128 & 95 & 33 & 0.295 \\
\hline No & 25 & 16 & 9 & \\
\hline \multicolumn{5}{|l|}{ Histology } \\
\hline $\begin{array}{l}\text { Squamous cell } \\
\text { carcinoma }\end{array}$ & 108 & 77 & 31 & 0.841 \\
\hline Adenocarcinoma & 26 & 20 & 6 & \\
\hline others & 19 & 14 & 5 & \\
\hline \multicolumn{5}{|l|}{ Histology grade } \\
\hline Well & 14 & 9 & 5 & 0.254 \\
\hline Moderate & 117 & 83 & 34 & \\
\hline Poor and Unknown & 22 & 19 & 3 & \\
\hline \multicolumn{5}{|l|}{ TNM stage } \\
\hline I & 18 & 6 & 12 & 0.001 \\
\hline II & 53 & 42 & 11 & \\
\hline III & 68 & 52 & 16 & \\
\hline IV & 14 & 11 & 3 & \\
\hline Metastasis & 111 & 87 & 24 & \\
\hline No metastasis & 42 & 24 & 18 & 0.009 \\
\hline
\end{tabular}

\section{Immunohistochemical evaluation}

A semiquantitative scoring criterion for IHC was used in which the staining intensity and positive areas were recorded as reported previously [23]. Briefly, the percentage of positive cells was determined by counting 500 cells in five randomly selected fields per section. The intensity of staining was scored on a 0-3 scale: zero (no staining), 1 (weak staining), 2 (moderate staining) and 3 (strong staining). The percentage of reactivity was scored as follows: 0 (no positive tumor cells), 1 ( $<10 \%$ positive tumor cells), 2 (10-50\% positive tumor cells) and 3 ( $>50 \%$ positive tumor cells). Next, the score was obtained by multiplying the intensity and reactivity rates. Scores of $<4$ suggested low expressions, and the remainder

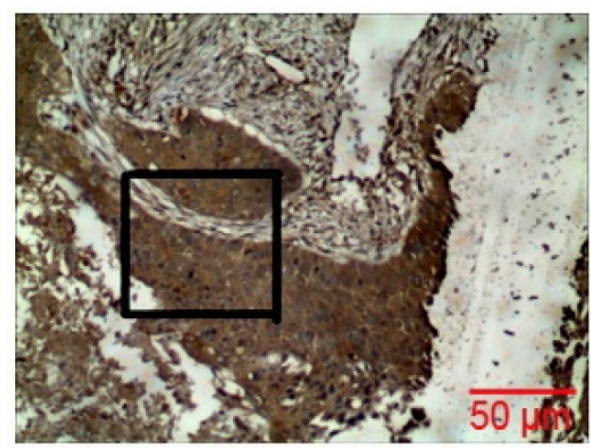

were classified as high expression. The immunostaining results were confirmed independently by two pathologists in a blinded manner.

\section{Statistical analysis}

Statistical procedures were analysed using the SPSS version 15.0 (SPSS, Chicago, IL, USA). The expression of Rac1 and EMT markers, and their correlation with clinicopathological parameters was analyzed using the chi-square test. Spearman's rank test was used to determine the correlation between Rac1 and EMT markers. Survival was estimated using the Kaplan-Meier method and compared with log-rank test. Univariate and multivariate analysis was conducted using Cox' regression model after adjusting for baseline characteristics. $\mathrm{P}<0.05$ was considered statistically significant.

\section{Results}

\section{Racl protein was highly expressed in lung cancer, and showed significant positive correlation with TNM stage and metastasis.}

Immunohistochemistry was used to detect Rac1 expression in lung cancer tissues. Rac1 was positively expressed mainly in the cytoplasm, and significantly expressed in lung cancer tissues (Fig. 1), with a positive expression rate of $72.55 \%$ (111/153). In tissues adjacent to lung cancer, the Rac1 expression was only $26.67 \%$ (12/45). Statistical analysis showed that Rac1 expression in the lung cancer tissue was significantly higher than in the adjacent tissue $\left(\mathrm{X}^{2}\right.$ =31.11, $\mathrm{P}=0.000$ ). Rac1 expression in lung cancer (Table 1) was not correlated with sex, age, smoking, pathological phenotype or degree of differentiation, but showed significant correlation with TNM stage $(\mathrm{P}=0.001)$ and metastasis $(\mathrm{P}=0.009)$.

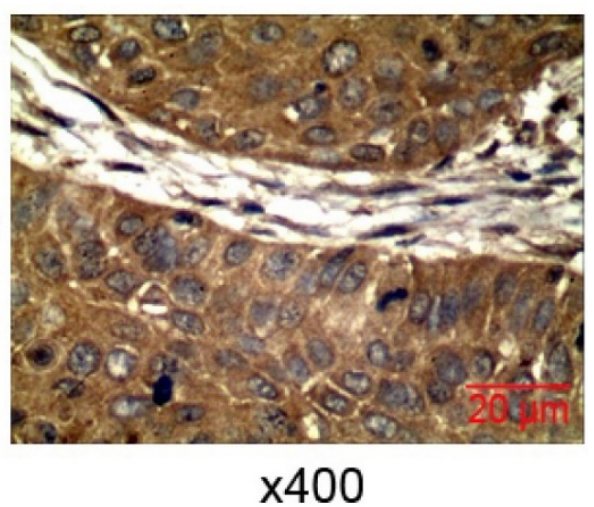

Figure 1. Expression of Racl in lung carcinoma tissues. IHC of Racl expression in lung carcinoma tissues (magnification $\times 100, \times 400$ ). 


\section{Racl expression correlated with poor prognosis}

Kaplan-Meier survival analysis showed that patients with high Rac1 expression had significantly shorter progression-free survival (PFS) and overall survival (OS) than those with low expression $(27.17 \pm 11.178$ vs. $19.00 \pm 1.717, \mathrm{P}=0.001 ; 49.51 \pm 10.520$ vs. 24.21 $\pm 2.482, \mathrm{P}=0.000$ ) (Fig. 2). Univariate and multivariate regression analysis showed (Table 2) that Rac1 expression was closely related to OS in lung cancer patients. The prognosis of patients with high Rac1 protein expression was significantly poorer than those with low expression ( $\mathrm{P}<0.001)$, and HR was 2.347 and 2.06, respectively. Univariate analysis indicated that higher TNM stage was associated with poorer prognosis $(\mathrm{P}=0.034, \mathrm{HR} 1.271)$. Patients with metastatic lung cancer showed poorer prognosis without any statistical significance $(\mathrm{P}=0.063)$. Other parameters were not correlated with OS. Rac1 is a potential prognostic marker of lung cancer.

\section{EMT markers were abnormally expressed in lung cancer tissues, and showed significant correlation with TNM stage and metastasis}

IHC was used to analyze the expression of EMT molecular markers such as Snail1, N-cad, Twist1, Vim, and E-cad in lung cancer tissues. Compared with normal tissues adjacent to lung cancer, the cancer tissues significantly expressed Snail1, N-cad, Twist1, and Vim, whereas E-cad was poorly expressed in most of the lung cancer tissues (Fig. 3). The positive expression rates were $86.27 \%(132 / 153)$, 79.08\%(121/153), 78.43\%(120/153), 61.44\%(94/153), $45.75 \%(70 / 153)(\mathrm{P}<0.05)$, respectively. The analysis of EMT and lung cancer clinicopathology showed that Snail1, N-cad, Twist1, Vim, and E-cad expression showed significant correlation with lung cancer TNM stage and metastasis. Twist1 expression showed significant correlation with smoking. E-cad expression was significantly correlated with differentiation of lung cancer. The EMT markers had no correlation with sex, age, smoking, pathological phenotype or degree of tumor differentiation (Table 3 ).
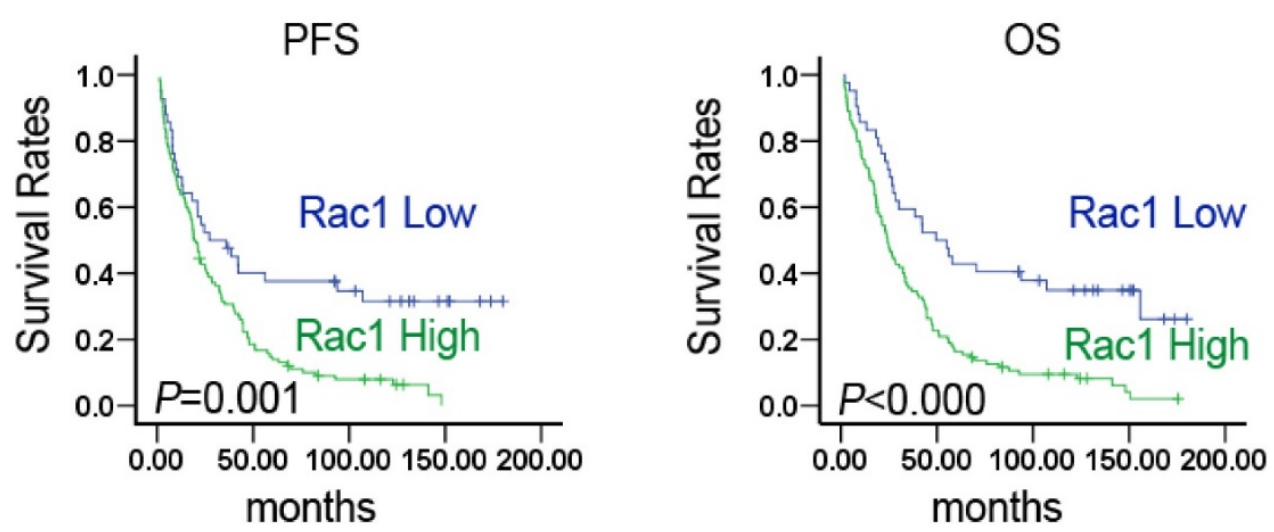

Figure 2. Racl expression was significantly correlated with survival in NSCLC. Kaplan-Meier estimated progression-free survival (PFS) and overall survival (OS) in NSCLC patients based on Racl expression pattern. P values were obtained using the log-rank test.

Table 2. Univariate and multivariate analysis of factors associated with overall survival in non-small cell lung cancer(NSCLC).

\begin{tabular}{|c|c|c|c|c|c|c|}
\hline \multirow[t]{2}{*}{ Variables } & \multicolumn{3}{|c|}{ Univariate analysis } & \multicolumn{3}{|c|}{ Multivariate analysis } \\
\hline & HR & $95 \% \mathrm{CI}$ & $\mathbf{P}$ & HR & $95 \% \mathrm{CI}$ & $\mathbf{P}$ \\
\hline Age $(\leq 60 />60$ years $)$ & 1.051 & $0.736-1.501$ & 0.784 & 0.712 & $0.328-1.546$ & 0.391 \\
\hline Gender (Female/Male) & 1.244 & $0.685-2.261$ & 0.473 & 1.239 & 0.505-3.037 & 0.640 \\
\hline Smoking (Yes/No) & 0.987 & $0.624-1.561$ & 0.955 & 0.922 & $0.474-1.792$ & 0.810 \\
\hline $\begin{array}{l}\text { Histology (Squamous cell } \\
\text { carcinoma/Adenocarcinoma/others) }\end{array}$ & 0.866 & $0.613-1.222$ & 0.413 & 0.776 & $0.552-1.090$ & 0.144 \\
\hline $\begin{array}{l}\text { Histology grade } \\
\text { (Well/Moderate/Poor and Unknown) }\end{array}$ & 1.323 & $0.895-1.957$ & 0.160 & 1.181 & $0.781-1.785$ & 0.431 \\
\hline Stage(I/II/III/IV) & 1.271 & $1.018-1.587$ & 0.034 & 1.166 & $0.908-1.497$ & 0.228 \\
\hline Metastasis(Yes/No) & 1.445 & $0.980-2.132$ & 0.063 & 1.246 & $0.798-1.947$ & 0.333 \\
\hline Rac1(High/Low) & 2.347 & $1.531-1.598$ & 0.000 & 2.206 & $1.413-3.444$ & 0.000 \\
\hline
\end{tabular}

HR: Hazard ratio; CI: confidence interval. 

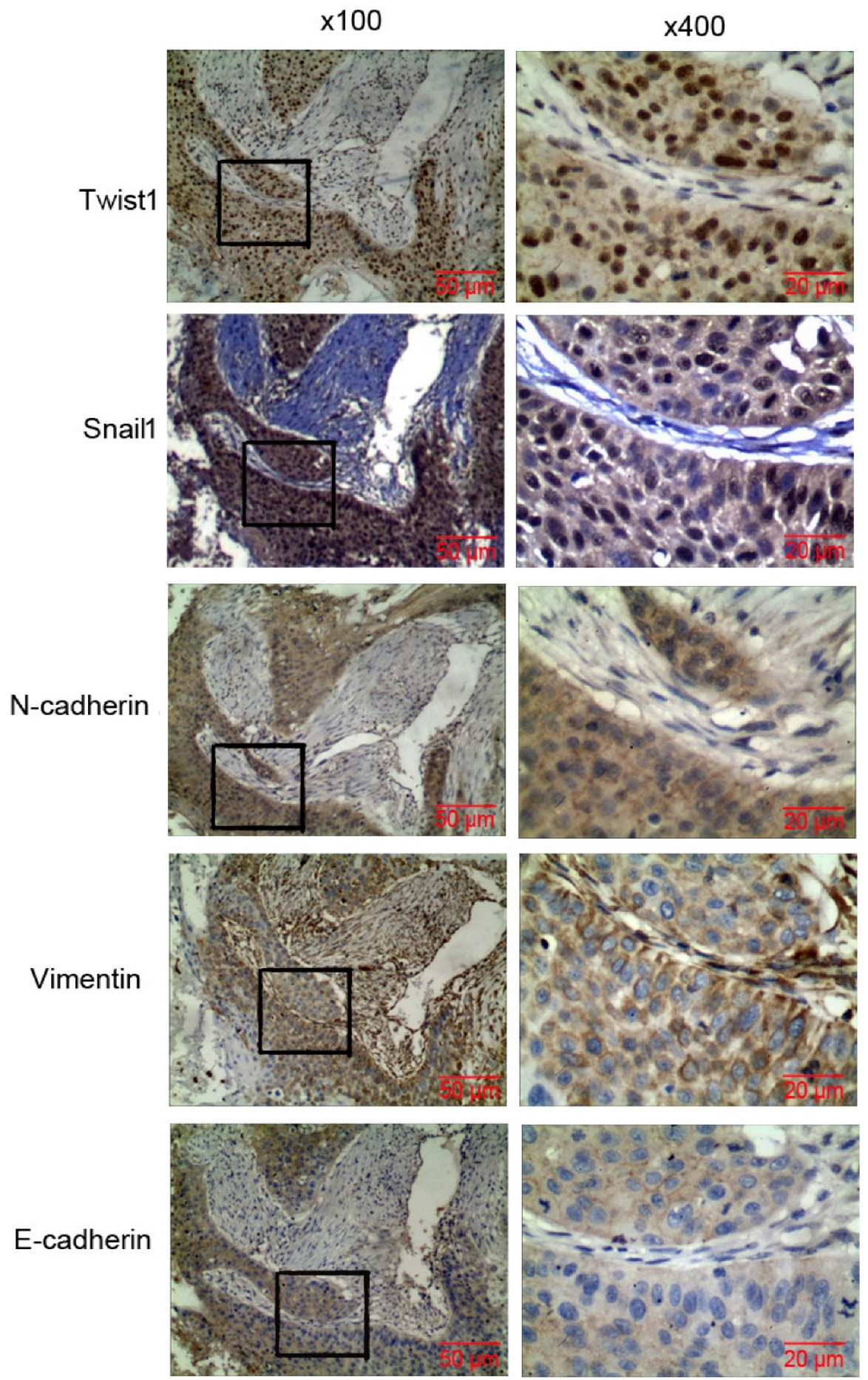

Figure 3. Expression of EMT markers in lung carcinoma tissues. IHC detection of the expression of Snaill, N-cad, Twistl, Vim, and E-cad in lung carcinoma tissues (magnification x 100, x 400).

\section{Abnormally expressed EMT molecular markers predicted poor clinical prognosis}

Kaplan-Meier survival analysis showed that the expression of Snail1, N-cad, Twist1, Vim, and E-cad was closely related to PFS and OS in lung cancer patients. Increased expression of Snail1, N-cad, Twist1, and Vim resulted in shorter PFS and OS. While E-cad was highly expressed, PFS and OS were significantly lengthened (Fig. 4). As shown in Table 4, multivariate regression analysis indicated that Snail1, $\mathrm{N}$-cad, Twist1, Vim, and E-cad expression was closely related to OS. The prognosis of patients with high Snail1, N-cad, Twist1, and Vim expression was significantly poor compared with those exhibiting low expression $(\mathrm{P}<0.05)$. Patients with low E-cad expression had significantly poorer prognosis 
compared with high E-cad expression $(\mathrm{P}<0.05)$. The results demonstrated that EMT molecular markers served as potential molecular markers for monitoring prognosis of lung cancer.

\section{Racl expression was positively correlated with Snail1, N-cad, Twist1, Vim expression, and negatively correlated with E-cad expression}

The correlation of Rac1 with EMT molecular markers was analyzed. The results indicated that Rac1 expression was significantly associated with the expression of EMT molecular markers. Rac1 expression showed a positive correlation with Snail1, $\mathrm{N}$-cad, Twist1, and Vim expression, and a negative correlation with E-cad expression (Table 5). In lung cancer tissues, Vim expression was negatively correlated with E-cad expression ( $\mathrm{r}=-0.487, \mathrm{P}<0.001)$. The results suggested that Rac1 promoted the progression, invasion, and metastasis or relapse of lung cancer by inducing EMT.

\section{Correlation of Racl and EMT expression with lung cancer radiosensitivity}

In order to explore the role of Rac1 and EMT in resistance to radiotherapy, a total of 153 lung cancer patients were divided into radiotherapy-sensitive and radiotherapy-resistant groups. As shown in Table 6, a high expression of Rac1 was closely related to resistant lung cancer. In the radiotherapy-resistant group, Rac1 was highly expressed, and in the sensitive group, Rac1 expression was lower $\left(X^{2}=24.950, P<0.001\right)$. The expression of EMT markers was also correlated with radiosensitivity. Compared with the sensitive group, the levels of Twist, Snail1, Vim, and $\mathrm{N}$-cad expression in the resistant group were significantly increased, and E-cad expression was significantly decreased. It indicated that increased Rac1 expression and altered EMT contributed to radioresistant lung cancer. Rac1 and EMT molecular markers represent predictive factors for radiotherapy-resistant lung cancer.

Table 3. Relationship between EMT phenotype and clinicopathologic characteristics.

\begin{tabular}{|c|c|c|c|c|c|c|c|c|c|c|c|c|c|c|c|c|}
\hline \multirow[t]{2}{*}{ Variable } & \multirow[t]{2}{*}{$\mathbf{N}$} & \multicolumn{3}{|c|}{ Snail1 } & \multicolumn{3}{|l|}{$\mathrm{N}$-cad } & \multicolumn{3}{|c|}{ Twist1 } & \multicolumn{3}{|l|}{ Vim } & \multicolumn{3}{|l|}{ E-cad } \\
\hline & & High & Low & $P$ Value & High & Low & $P$ Value & High & Low & $P$ Value & High & Low & $P$ Value & High & Low & $P$ Value \\
\hline \multicolumn{17}{|l|}{ Age (years) } \\
\hline$\leq 60$ & 57 & 49 & 8 & 0.932 & 45 & 12 & 0.974 & 45 & 12 & 0.905 & 36 & 21 & 0.736 & 27 & 30 & 0.757 \\
\hline$>60$ & 96 & 83 & 13 & & 76 & 20 & & 75 & 21 & & 58 & 38 & & 43 & 53 & \\
\hline \multicolumn{17}{|l|}{ Gender } \\
\hline male & 141 & 122 & 19 & 0.758 & 111 & 30 & 0.706 & 108 & 33 & 0.058 & 85 & 56 & 0.315 & 65 & 76 & 0.767 \\
\hline female & 12 & 10 & 2 & & 10 & 2 & & 12 & 0 & & 9 & 3 & & 5 & 7 & \\
\hline \multicolumn{17}{|l|}{ Smoking } \\
\hline Yes & 128 & 113 & 15 & 0.103 & 101 & 27 & 0.902 & 96 & 32 & 0.020 & 75 & 53 & 0.102 & 62 & 66 & 0.131 \\
\hline No & 25 & 19 & 6 & & 20 & 5 & & 24 & 1 & & 19 & 6 & & 8 & 17 & \\
\hline \multicolumn{17}{|l|}{ Histology } \\
\hline Squamous cell carcinoma & 108 & 93 & 15 & 0.916 & 86 & 21 & 0.715 & 82 & 26 & 0.396 & 62 & 46 & 0.271 & 50 & 58 & 0.111 \\
\hline Adenocarcinoma & 26 & 23 & 3 & & 19 & 7 & & 21 & 5 & & 18 & 8 & & 15 & 11 & \\
\hline others & 19 & 16 & 3 & & 15 & 4 & & 17 & 2 & & 14 & 5 & & 5 & 14 & \\
\hline \multicolumn{17}{|l|}{ Histology grade } \\
\hline Well & 14 & 14 & 0 & 0.263 & 13 & 1 & 0.413 & 11 & 3 & 0.106 & 7 & 7 & 0.085 & 9 & 5 & 0.03 \\
\hline Moderate & 117 & 98 & 19 & & 91 & 26 & & 88 & 29 & & 69 & 48 & & 58 & 59 & \\
\hline Poor and Unknown & 22 & 10 & 2 & & 17 & 5 & & 21 & 1 & & 18 & 4 & & 3 & 19 & \\
\hline \multicolumn{17}{|l|}{ TNM stage } \\
\hline I & 18 & 10 & 8 & 0.010 & 9 & 9 & 0.010 & 9 & 9 & 0.020 & 4 & 14 & 0.002 & 13 & 5 & 0.012 \\
\hline II & 53 & 47 & 6 & & 44 & 9 & & 44 & 9 & & 32 & 21 & & 28 & 25 & \\
\hline III & 68 & 61 & 7 & & 55 & 13 & & 56 & 12 & & 48 & 20 & & 26 & 42 & \\
\hline IV & 14 & 14 & 0 & & 13 & 1 & & 11 & 3 & & 10 & 4 & & 3 & 11 & \\
\hline Metastasis & 111 & 101 & 10 & 0.006 & 94 & 17 & 0.006 & 93 & 18 & 0.009 & 76 & 35 & 0.004 & 45 & 66 & 0.035 \\
\hline No metastasis & 42 & 31 & 11 & & 27 & 15 & & 27 & 15 & & 18 & 24 & & 25 & 17 & \\
\hline
\end{tabular}

Table 4. Cox regression analyses of EMT markers associated with OS in NSCLC.

\begin{tabular}{|c|c|c|c|c|c|c|}
\hline \multirow[t]{2}{*}{ Variables } & \multicolumn{3}{|c|}{ Univariate analysis } & \multicolumn{3}{|c|}{ Multivariate analysis } \\
\hline & HR & $95 \% \mathrm{CI}$ & $\mathrm{P}$ & HR & $95 \% \mathrm{CI}$ & $\mathrm{P}$ \\
\hline Snail1 & 2.262 & $1.291-3.962$ & 0.004 & 2.117 & $1.181-3.793$ & 0.012 \\
\hline N-cad & 1.949 & $1.243-3.056$ & 0.004 & 1.867 & $1.167-2.988$ & 0.009 \\
\hline Twist1 & 2.171 & $1.388-3.396$ & 0.001 & 2.076 & $1.304-3.303$ & 0.002 \\
\hline Vim & 1.885 & $1.314-2.706$ & 0.001 & 1.776 & $1.209-2.579$ & 0.003 \\
\hline E-cad & 0.506 & $0.354-0.724$ & 0.000 & 0.518 & $0.352-0.763$ & 0.001 \\
\hline
\end{tabular}

HR: Hazard ratio; $\mathrm{CI}$ : confidence interval. 

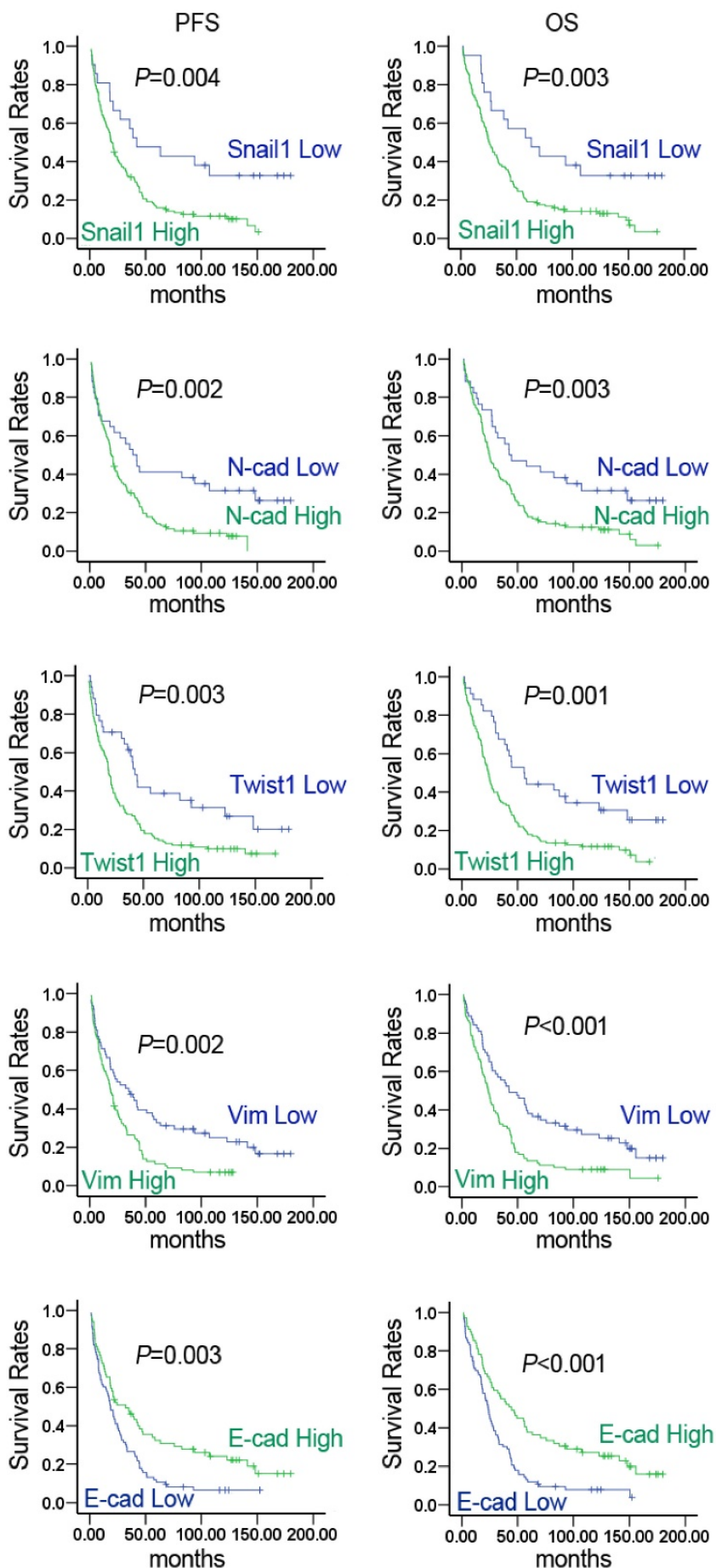

Figure 4. Expression of EMT markers was significantly correlated with survival of NSCLC patients. Kaplan-Meier curves for estimation of progression-free survival (PFS) and overall survival (OS) in NSCLC patients based on the expression levels of EMT markers. P values were obtained using the log-rank test.

\section{Discussion}

In our study, we found that Rac1 and EMT mesenchymal markers Snail1, Twist1, N-cadherin, and Vimentin were highly expressed in lung cancer tissues. The EMT epithelial marker E-cadherin was poorly expressed. Rac1 protein expression showed positive correlation with Snail1, Twist1, N-cadherin,
Vimentin expression and negative correlation with E-cadherin expression. The abnormal expression of Rac1 and EMT molecular markers was closely related to TNM stage, metastasis and prognosis. Furthermore, the elevated expression of Rac1, Vimentin, N-cadherin, Twist1 and Snail1, and suppression of E-cadherin expression were positively correlated with radiotherapy resistance. 
Table 5. Correlation between Racl expression and EMT markers.

\begin{tabular}{|c|c|c|c|c|c|}
\hline \multirow[t]{2}{*}{ EMT markers } & \multirow[t]{2}{*}{$\mathbf{N}$} & \multicolumn{2}{|c|}{ Rac1 } & \multirow[t]{2}{*}{$\mathbf{r}$} & \multirow[t]{2}{*}{$\mathbf{P}$} \\
\hline & & High expression & Low expression & & \\
\hline Snail1 & & 111 & 42 & 0.563 & 0.000 \\
\hline High expression & 132 & 109 & 23 & & \\
\hline Low expression & 21 & 2 & 19 & & \\
\hline N-cad & & & & 0.440 & 0.000 \\
\hline High expression & 121 & 100 & 21 & & \\
\hline Low expression & 32 & 11 & 21 & & \\
\hline Twist1 & & & & 0.247 & 0.002 \\
\hline High expression & 120 & 94 & 26 & & \\
\hline Low expression & 33 & 17 & 16 & & \\
\hline Vim & & & & 0.536 & 0.000 \\
\hline High expression & 94 & 86 & 8 & & \\
\hline Low expression & 59 & 25 & 34 & & \\
\hline E-cad & & & & -0.464 & 0.000 \\
\hline High expression & 70 & 35 & 35 & & \\
\hline Low expression & 83 & 76 & 7 & & \\
\hline
\end{tabular}

Table 6. Expression of Racl, Twist1, Snaill, Vimentin, $\mathrm{N}$-cadherin, and $\mathrm{E}$-cadherin in radioresistant and radiosensitive NSCLC.

\begin{tabular}{|c|c|c|c|c|c|}
\hline & $\mathrm{N}$ & High & Low & $X^{2}$ & $P$ Value \\
\hline \multicolumn{6}{|l|}{ Rac1 } \\
\hline Radiosensitivity & 54 & 26 & 28 & 24.950 & 0.000 \\
\hline Radioresistance & 99 & 85 & 14 & & \\
\hline \multicolumn{6}{|c|}{ E-cadherin } \\
\hline Radiosensitivity & 54 & 14 & 40 & 13.216 & 0.000 \\
\hline Radioresistance & 99 & 56 & 43 & & \\
\hline \multicolumn{6}{|c|}{ Twist } \\
\hline Radiosensitivity & 54 & 34 & 20 & 11.804 & 0.001 \\
\hline Radioresistance & 99 & 86 & 13 & & \\
\hline \multicolumn{6}{|c|}{ Snail } \\
\hline Radiosensitivity & 54 & 40 & 14 & 10.490 & 0.001 \\
\hline Radioresistance & 99 & 92 & 7 & & \\
\hline \multicolumn{6}{|c|}{ Vimentin } \\
\hline Radiosensitivity & 54 & 26 & 28 & 6.221 & 0.013 \\
\hline Radioresistance & 99 & 68 & 31 & & \\
\hline \multicolumn{6}{|c|}{ N-cadherin } \\
\hline Radiosensitivity & 54 & 36 & 18 & 7.781 & 0.005 \\
\hline Radioresistance & 99 & 85 & 14 & & \\
\hline
\end{tabular}

Rac GTP enzyme (Rho protein family member) regulated EMT of tumor cells and promoted tumor metastasis [12]. The Rac GTP enzyme sub-family includes Rac1, Rac2, Rac3 and Rac1b (spliced variant of Rac1). Rac1 has been the main focus of recent studies. Rac1 is abnormally expressed and activated in stomach and ovarian cancers. The high expression of Rac1 is positively correlated with highly invasive cancer and poor prognosis [17, 18, 25]. We found that Rac1 was significantly expressed in lung cancer tissues, and was positively correlated with TNM stage, metastasis and poor prognosis, which was consistent with Yuan K's report [26]. Knockdown of Rac1 expression or inhibition of Rac1 signal significantly attenuated the invasion and migration of lung cancer cells $[19,27]$. Therefore, up-regulation of Rac1 expression may promote lung cancer initiation and progression. Rac1 is a potential molecular marker associated with lung cancer prognosis. It represents a potential molecular target for the prevention and cure of lung cancer.

Numerous studies suggest that metastasis is one of the most decisive factors influencing patient prognosis. Current studies suggest that EMT broadly regulates cancer metastasis [28, 29]. Reduced E-cadherin expression increases the expression of Vimentin, N-cadherin, Snail1, and Twist1. The study showed that high expression of Rac1 induced EMT in tumor cells in vitro and in vivo [17, 18], and promoted invasion and metastasis of tumor. The inhibition of Rac1 expression significantly up-regulated the expression of E-cadherin, down-regulated the expression of $\mathrm{N}$-cadherin and Vimentin, and significantly inhibited EMT in cancer cells [21]. However, the correlation between Rac1 and EMT is still unclear in lung cancer tissues. Therefore, IHC was used to analyze the expression of EMT molecular markers including Snail1, N-cadherin, Twist1, Vimentin, and E-cadherin in lung cancer tissues, and correlate their levels with Rac1 expression. The results showed that Snail1, N-cadherin, Twist1, and Vimentin were highly expressed, and E-cadherin was poorly expressed. The abnormal expression of EMT molecular markers was closely related to TNM stage, metastasis and prognosis. Rac1 expression was positively correlated with Snail1, Twist1, N-cadherin and Vimentin expression, and negatively correlated with E-cadherin expression. The result suggested that EMT molecular markers can be used as potential molecular markers for lung cancer prognosis, and Rac1 improved the progression, invasion, metastasis or relapse of lung cancer by inducing EMT in lung cancer cells.

The results indicated that EMT not only played a key role in tumor invasion and metastasis, but also was closely related with tolerance of tumor cell therapy $[4,30]$. Tumor cells resistant to radiotherapy displayed EMT characteristics, resulting in tumor invasion and metastasis [10, 31]. Ho et al. [32] reported that radiotherapy increased the invasion and EMT of remaining tumor cells via STAT3-mediated BCL-XL expression. The study indicated that EMT was closely related to radiotherapy resistance in lung cancer [33]. Knocking down the expression of lung cancer cell line TBK1 significantly down-regulated the transcriptional activation of ZEB1 and reversed EMT induced by ionizing radiation, which further increased the radiotherapy sensitivity of lung tumor cells [34]. Abnormal Rac1 expression and activation were related to radiotherapy resistance of various tumors. Skvortsov et al. reported that Rac1 was a potential marker of radiotherapy resistance in head 
and neck cancer [35]. Rac1 was significantly expressed and activated in radiotherapy resistant head and neck squamous cell carcinoma, decreasing the activation of Rac1 cell migration and invasion [36]. Yan et al. [37] found that Rac1 was the key molecule activated in $\mathrm{G}_{2} / \mathrm{M}$ of breast cancer induced by $\gamma$ rays, increasing the tolerance to gamma radiation. Rac1 plays a key role in the acquired resistance of pancreatic cancer to ionizing radiation. Treatment with Rac1-specific inhibitors or Rac1 signal blockers significantly increased the sensitivity of pancreatic cancer to ionizing radiation [38]. Therefore, the relationship between Rac1 expression, EMT and sensitivity of lung cancer radiation was analyzed. The results showed that Rac1, Twist1, Snail1, Vimentin, N-cadherin were highly expressed in lung cancer patients with radiotherapy resistance, and E-cadherin was poorly expressed. Rac1 and EMT predict resistance to radiotherapy of lung cancer. The highly expressed Rac1 and altered EMT contribute to resistance. The mechanisms underlying the resistance need further elucidation.

In summary, our results suggest that Rac1 and EMT protein expression levels are associated with cancer progression, metastasis and resistance to radiotherapy. Rac1 may promote NSCLC progression and metastasis via EMT, which may be considered a potential therapeutic target in NSCLC. However, large prospective studies are required to further confirm our findings.

\section{Abbreviations}

Rac1: Ras-related C3 botulinum toxin substrate1, EMT: epithelialmesenchymal transition, N-cad: $\mathrm{N}$-cadherin, E-cad: E-cadherin, Vim: Vimentin, NSCLC: non-small cell lung cancer, PFS: progression-free survival, OS: overall survival.

\section{Ethics Committee Approval and Patient Consent}

This study was approved by the Joint Ethics Committee of the Hunan Cancer Hospital and The Affiliated Cancer Hospital of Xiangya School of Medicine, Central South University Health Authority in China. All the individuals participating in this project signed informed consent.

\section{Acknowledgement}

This work was supported in part by grants fromthe following sources: the National Natural Science Foundation of China (81472595, 81402006); the Research Project of Health and Family Planning Commission of Hunan Province(B2016045); the Natural Science Foundation of Hunan Province (2015JJ2094); Development and Reform Commission of Hunan Province; the Science Foundation of Hunan Cancer Hospital (A2013-01)

\section{Competing Interests}

The authors have declared that no competing interest exists.

\section{References}

1. Siegel RL, Miller KD, Jemal A. Cancer statistics, 2015. CA Cancer J Clin. 2015; 65: 5-29.

2. Torre LA, Siegel RL, Jemal A. Lung Cancer Statistics. Adv Exp Med Biol. 2016; 893: 1-19.

3. DeSantis CE, Lin CC, Mariotto AB, et al. Cancer treatment and survivorship statistics, 2014. CA Cancer J Clin. 2014; 64: 252-71.

4. Smith BN, Bhowmick NA. Role of EMT in Metastasis and Therapy Resistance. J Clin Med. 2016; 5.

5. Seton-Rogers S. Epithelial-mesenchymal transition: Untangling EMT's functions. Nat Rev Cancer. 2016; $16: 1$.

6. $\mathrm{Vu} \mathrm{T}$, Jin L, Datta PK. Effect of Cigarette Smoking on Epithelial to Mesenchymal Transition (EMT) in Lung Cancer. J Clin Med. 2016; 5.

7. Beck TN, Chikwem AJ, Solanki NR, et al. Bioinformatic approaches to augment study of epithelial-to-mesenchymal transition in lung cancer. Physiol Genomics. 2014; 46: 699-724.

8. Sato M, Shames DS, Hasegawa Y. Emerging evidence of epithelial-to-mesenchymal transition in lung carcinogenesis. Respirology. 2012; 17: 1048-59.

9. Zhang P, Sun Y, Ma L. ZEB1: at the crossroads of epithelial-mesenchymal transition, metastasis and therapy resistance. Cell Cycle. 2015; 14: 481-7.

10. Moncharmont C, Levy A, Guy JB, et al. Radiation-enhanced cell migration/invasion process: a review. Crit Rev Oncol Hematol. 2014; 92: 133-42.

11. Zhang J, Tian XJ, Xing J. Signal Transduction Pathways of EMT Induced by TGF-beta, SHH, and WNT and Their Crosstalks. J Clin Med. 2016; 5.

12. Leve F, Morgado-Diaz JA. Rho GTPase signaling in the development of colorectal cancer. J Cell Biochem. 2012; 113: 2549-59.

13. Xiang S, Li M, Xie X, et al. Rapamycin Inhibits Epithelial-to-Mesenchymal Transition of Peritoneal Mesothelium Cells through Regulation of RhoGTPases. FEBS J. 2016.

14. Su B, Su J, Zeng Y, et al. Diallyl disulfide suppresses epithelial-mesenchymal transition, invasion and proliferation by downregulation of LIMK1 in gastric cancer. Oncotarget. 2016; 7: 10498-512.

15. Wertheimer E, Gutierrez-Uzquiza A, Rosemblit C, et al. Rac signaling in breast cancer: a tale of GEFs and GAPs. Cell Signal. 2012; 24: 353-62.

16. Huang $\mathrm{X}$, Wei $\mathrm{Y}, \mathrm{Ma} \mathrm{H}$, et al. Vitreous-induced cytoskeletal rearrangements via the Rac1 GTPase-dependent signaling pathway in human retinal pigment epithelial cells. Biochem Biophys Res Commun. 2012; 419: 395-400.

17. Bid HK, Roberts RD, Manchanda PK, et al. RAC1: an emerging therapeutic option for targeting cancer angiogenesis and metastasis. Mol Cancer Ther. 2013; 12: 1925-34.

18. Leng $\mathrm{R}$, Liao $\mathrm{G}$, Wang $\mathrm{H}$, et al. Rac1 expression in epithelial ovarian cancer: effect on cell EMT and clinical outcome. Med Oncol. 2015; 32: 329.

19. Nie F, Wang XF, Zhao SY, et al. Gene silencing of Rac1 with RNA interference mediated by ultrasound and microbubbles in human LoVo cells: evaluation of cell invasion inhibition and metastatic. J Drug Target. 2015; 23: 380-6.

20. Wang XC, Du LQ, Tian LL, et al. Expression and function of miRNA in postoperative radiotherapy sensitive and resistant patients of non-small cell lung cancer. Lung Cancer. 2011; 72:92-9.

21. Niu H, Wu B, Jiang $\mathrm{H}$, et al. Mechanisms of RhoGDI2 mediated lung cancer epithelial-mesenchymal transition suppression. Cell Physiol Biochem. 2014; 34: 2007-16.

22. Meng Q, Sun W, Li M, et al. Increased Expression of Eps15 Homology Domain 1 is Associated with Poor Prognosis in Resected Small Cell Lung Cancer. J Cancer. 2015; 6: 990-5.

23. Zhou Y, Liao Q, Li X, et al. HYOU1, Regulated by LPLUNC1, Is Up-Regulated in Nasopharyngeal Carcinoma and Associated with Poor Prognosis. J Cancer. 2016; 7: 367-76.

24. Rathinam R, Berrier A, Alahari SK. Role of Rho GTPases and their regulators in cancer progression. Front Biosci (Landmark Ed). 2011; 16: 2561-71.

25. Ji J, Feng X, Shi M, et al. Rac1 is correlated with aggressiveness and a potential therapeutic target for gastric cancer. Int J Oncol. 2015; 46: 1343-53.

26. Yuan K, Qian C, Zheng R. Prognostic significance of immunohistochemical Rac1 expression in survival in early operable non-small cell lung cancer. Med Sci Monit. 2009; 15: BR313-9.

27. Chen QY, Xu LQ, Jiao DM, et al. Silencing of Rac1 modifies lung cancer cell migration, invasion and actin cytoskeleton rearrangements and enhances chemosensitivity to antitumor drugs. Int J Mol Med. 2011; 28: 769-76.

28. Hanahan D, Weinberg RA. Hallmarks of cancer: the next generation. Cell. 2011; 144: 646-74. 
29. Shan ZZ, Yan XB, Yan LL, et al. Overexpression of Tbx3 is correlated with Epithelial-Mesenchymal Transition phenotype and predicts poor prognosis of colorectal cancer. Am J Cancer Res. 2015; 5: 344-53.

30. Nantajit D, Lin D, Li JJ. The network of epithelial-mesenchymal transition: potential new targets for tumor resistance. J Cancer Res Clin Oncol. 2015; 141: 1697-713.

31. Chang L, Graham PH, Hao J, et al. Emerging roles of radioresistance in prostate cancer metastasis and radiation therapy. Cancer Metastasis Rev. 2014; 33: 469-96.

32. Ho JN, Kang GY, Lee SS, et al. Bcl-XL and STAT3 mediate malignant actions of gamma-irradiation in lung cancer cells. Cancer Sci. 2010; 101: 1417-23.

33. Yang HJ, Kim N, Seong KM, et al. Investigation of radiation-induced transcriptome profile of radioresistant non-small cell lung cancer A549 cells using RNA-seq. PLoS One. 2013; 8: e59319.

34. Liu W, Huang YJ, Liu C, et al. Inhibition of TBK1 attenuates radiation-induced epithelial-mesenchymal transition of A549 human lung cancer cells via activation of GSK-3beta and repression of ZEB1. Lab Invest. 2014; 94: 362-70.

35. Skvortsov S, Dudas J, Eichberger P, et al. Rac1 as a potential therapeutic target for chemo-radioresistant head and neck squamous cell carcinomas (HNSCC). Br J Cancer. 2014; 110: 2677-87.

36. Skvortsov S, Jimenez CR, Knol JC, et al. Radioresistant head and neck squamous cell carcinoma cells: intracellular signaling, putative biomarkers for tumor recurrences and possible therapeutic targets. Radiother Oncol. 2011; 101: 177-82.

37. Yan Y, Greer PM, Cao PT, et al. RAC1 GTPase plays an important role in gamma-irradiation induced G2/M checkpoint activation. Breast Cancer Res. 2012; 14: R60.

38. Yan Y, Hein AL, Etekpo A, et al. Inhibition of RAC1 GTPase sensitizes pancreatic cancer cells to gamma-irradiation. Oncotarget. 2014; 5: 10251-70. 